\title{
Indications for Referral for Pediatric Rhinosinusitis
}

\author{
John W. Frederick, MD \\ Jeffrey D. Suh, $M D^{*}$
}

\author{
Address \\ *Department of Head and Neck Surgery, David Geffen School of Medicine at UCLA, \\ 10833 Le Conte Ave, CHS 62-132, Los Angeles, CA 90095-1624, USA \\ Email: JeffSuh@mednet.ucla.edu
}

Published online: 22 July 2015

(C) Springer International Publishing AG 2015

This article is part of the Topical Collection on Otolaryngology

Keywords Rhinosinusitis · Chronic sinusitis - Pediatric sinusitis - Endoscopic sinus surgery

\section{Opinion statement}

Rhinosinusitis in children is defined as inflammation of the nose and paranasal sinuses (Fokkens et al. Rhinology. 2012;50:1-12). Pediatric chronic rhinosinusitis (CRS) is relatively uncommon compared to acute rhinosinusitis (ARS) and is characterized by sinus symptoms lasting over 12 weeks despite medical therapy. Pathogenesis of this disease is multifactorial and generally involves an initial insult (usually a viral sinusitis) followed by bacterial seeding and mucosal inflammation. The diagnosis of chronic rhinosinusitis in the pediatric population can be challenging as symptoms can be very similar to common conditions such as allergic rhinitis or adenoiditis. In many cases, objective findings such as inflammation seen on nasal endoscopy or sinus CT scans are necessary to confirm the diagnosis of CRS. The mainstay of treatment of pediatric CRS is medical therapy with surgical therapy reserved for the minority of patients. Standard medical therapy usually includes a combination of nasal corticosteroid sprays, nasal irrigation, and decongestants. Antibiotics are usually reserved for bacterial acute exacerbations of CRS and usually target the most common offending pathogens (Streptococcus pneumoniae, Haemophilus influenzae, and Moraxella catarrhalis). Referral to an otolaryngologist is recommended for children who fail medical therapy, have orbital complications of sinusitis, or require surgery. There are currently many alternate medical treatment options available to physicians; however, data supporting their efficacy is lacking in many cases. Recent studies show that even minimally invasive surgical procedures, such as adenoidectomy and/or balloon sinuplasty can lead to significant symptom improvement. Further, traditional endoscopic sinus surgery offers a safe, highly effective treatment option when necessary.

\section{Introduction}

Rhinosinusitis in children is a common disease that infection. The classification of rhinosinusitis revolves usually occurs in the context of a viral upper respiratory 
acute (less than 4 weeks), subacute (4-12 weeks), or chronic (more than 12 weeks, with or without acute exacerbations) $[1,2 \bullet \bullet]$. Unlike acute rhinosinusitis, pediatric chronic rhinosinusitis (pCRS) is a complex disease with a pathophysiology that usually results from multiple genetic and environmental factors that if untreated, can have substantial effects on quality of life. The diagnosis and management of pediatric CRS was previously based on guidelines designed for adults, but an updated consensus statement has been recently published by the American Academy of Otolaryngology-Head and Neck Surgery (AAO-HNS) in 2014 [2••]. CRS in children is now defined as an inflammation of the nose and paranasal sinuses lasting over 12 weeks characterized by two or more symptoms (Table 1). These guidelines also require objective evidence of sinonasal inflammation, either by nasal endoscopy or by computed tomography (CT) of the sinuses to differentiate CRS from other chronic nasal conditions [3-5].

\section{Development of the paranasal sinuses}

An understanding of the development of the paranasal sinuses is of great importance not only in the diagnosis or pCRS but also in the treatment. For example, management of CRS in children under the age of 12 can be distinctly different than those ranged from children 13-18 years old due to differences in sinus development. The embryology of the sinuses is a complex process that involves fusion and involution of the medial extensions from the lateral wall of the nasal capsule, the ethmoturbinals. Beginning at the 8th week of development, these turbinals form the normal structures of the nasal anatomy and lay the groundwork for mucociliary clearance. The first ethmoturbinal will eventually regress to form both the agger nasi (the anterior most ethmoid air cell) and the uncinate process. The second ethmoturbinal develops into the middle turbinate and ethmoid bulla, while the third turbinal will eventually develop into the superior turbinate [6-9].

The majority of all sinuses will reach adult size by the age of 15 years with the frontal sinus, the last to develop, reaching adult size by 19 years. Of all the sinuses, the maxillary and ethmoid sinuses are most developed at birth. They tend to exhibit early growth, reaching near adult size by the age of 10 [10]. The sphenoid sinuses are generally appreciable on imaging before 3 years of age and become fully developed by age 12-14 [11, 12]. Because the sinuses are generally developed by adolescence, the current AAO-HNS guidelines on pCRS state that children 13 years of age or older can typically be managed like adults $[2 \bullet \bullet]$.

\section{The microbiology of acute and chronic pediatric sinusitis}

In general, most cases of pediatric acute rhinosinusitis develop after a viral upper respiratory infection. Bacterial sinusitis occurs much less commonly, in only 0.5 to $2 \%$ of cases, and usually as a complication of viral sinusitis [13]. Viral URIs are usually characterized by nasal symptoms (discharge, congestion, and obstruction) or cough. Fever, tends to occur early in the illness, usually with other constitutional symptoms such as headache and myalgias. The course of most viral URIs is 5-7 days, with peak symptoms generally occurring by days $2-3$ [14, 
Table 1. Definition of chronic rhinosinusitis. Adapted from Brietzke et al. [2••]

Pediatric chronic rhinosinusitis

$>12$ weeks of two or more of the following symptoms

- Nasal blockage/obstruction/congestion

- Nasal discharge (anterior or posterior drip)

- Facial pain/pressure

- Cough

AND inflammation documented by one or more of the following findings:

- Purulent mucus or edema in the middle meatus or ethmoid region

- Polyps in the nasal cavity or middle meatus

- Radiographic imaging showing inflammation of the paranasal sinuses

15]. During acute infections, viruses induce the release of multiple inflammatory cytokines and mediators in the affected sinonasal mucosa. These substances, such as IL- 8 and bradykinin, induce inflammation, obstruction, and derangement of normal mucociliary clearance. With obstruction of mucus flow comes the possibility for bacterial infection. Similar to the bacteriology of otitis media, Streptococcus pneumoniae, Haemophilus influenzae, and Moraxella catarrhalis account for more than $70 \%$ of the bacteria identified in acute bacterial rhinosinusitis [16, 17]. Anaerobic organisms are found in $6 \%$ of cultures, with a higher prevalence in patients with chronic rhinosinusitis. Detailing the pathogens behind the development of pCRS is more difficult, perhaps due to decreased bacterial concentration from previous antibiotic treatments. In review of 295 maxillary sinus cultures in children with CRS, Hsin et al. found the most common bacteria to be $\alpha$-hemolytic Streptococcus, H. influenza, S. pneumoniae, and coagulase negative Staphylococcus [18].

Recently, there has been interest in the role of biofilms in the development of pCRS. A biofilm is a complete microenvironment of organisms with complex cellular interactions, genetic diversity, and extracellular glycoconjugates that, in combination, are thought to provide an environment for improvement organism survival and antibiotic resistance [19-21]. Many of the bacteria identified in pCRS are capable of forming biofilms, and the presence of biofilms has been well-

documented adult CRS [22]. It is believed that biofilms shed bacteria which contribute to recurrent exacerbations of CRS [20, 23]. This continuous seeding allows for perpetual inflammation, infection, and obstruction of the sinuses and the resilience of pCRS [24].

When the pediatric population is considered, an important structural difference in the development of pCRS is the abundance of adenoid tissue. The adenoids are in close proximity to the paranasal sinuses, and studies have found similar microbiomes $[25 \bullet \bullet, 24]$. Adenoiditis is considered to be an important contributing factor to pCRS, especially in younger children, and are thought to

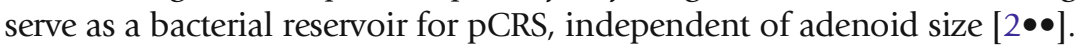
Bacterial biofilms have been identified in over $88 \%$ of adenoidectomy specimens from children with CRS, in contrast to adenoids removed for patients with sleep apnea (0-6.5\%). Adenoid biofilms can explain resistance to antibiotic treatment and improvement after surgery. The AAO-HNS guidelines 
recommended adenoidectomy as the initial surgical therapy for patients aged up to 6 years; however, less consensus was obtained for patients age 6-12 years $[2 \bullet \bullet]$.

\section{Complications of acute rhinosinusitis}

Complications of pediatric acute rhinosinusitis (ARS) are seen once in every 95,000 admissions [26]. The pathogenesis of the most frequently encountered complications revolves around the extension or involvement of adjacent structures. Orbital or periorbital infection is the most common complication seen with ARS [27], and either of these complications is an absolute indication for immediate referral to a trained otolaryngologist. As detailed by Chandler et al., any infection involving the orbit can be graded based on location and severity regardless of cause [28] (Table 2). Class I includes preseptal cellulitis and patients will not have limitation of eye movement or visual impairment. Figure 1 illustrates a case of preseptal cellulitis due to an acute sinus infection. Class II is described as orbital cellulitis and is the earliest stage exhibiting limitation of extra ocular movements. The patient will have diffuse edema of adipose tissues but no abscess formation. Class III patients have subperiosteal abscess. Class IV shows extension of the abscess to the orbit, and class $V$ patients exhibit cavernous sinus thrombosis. Considering the possible intracranial extension and life-threatening nature of these infections, current guidelines recommend aggressive treatment with intravenous antibiotics, decongestants, and occasional surgical intervention. Referral and evaluation by an otolaryngologist for any complicated sinus infection is indicated due to a potential for rapid progression of disease even with antibiotic therapy. However, it has been shown that even in the setting of a subperiosteal abscess (Chandler class III), successful treatment can be accomplished in $93 \%$ of children with medical management alone [29, 30]. Medical management is most successful for Chandler class I or early class II, but all pediatric patients with acute sinusitis with orbital involvement require close observation by an otolaryngologist for possible surgical drainage of the sinuses.

Meningitis, epidural, subdural, and brain abscess represent the most common intracranial complications seen with ARS. The most common of these complications, subgaleal and epidural abscesses, are often associated with

\section{Table 2. Chandler's classification of orbital infection}

\begin{tabular}{|c|c|c|}
\hline Stage & Diagnosis & Clinical features \\
\hline I & Preseptal/periorbital cellulitis & $\begin{array}{l}\text { Inflammatory upper eyelid edema. The lid will be nontender without } \\
\text { vision impairment or limitation of extraocular movements }\end{array}$ \\
\hline II & Postseptal/orbital cellulitis & $\begin{array}{l}\text { The development of periorbital edema, proptosis, chemosis, with or } \\
\text { without limitation of extraocular movements. }\end{array}$ \\
\hline III & Subperiosteal abscess & $\begin{array}{l}\text { Marked by a severe limitation of extraocular movements and a decrease } \\
\text { in visual acuity. }\end{array}$ \\
\hline IV & Orbital abscess & $\begin{array}{l}\text { Continued progression of proptosis, complete ophthalmoplegia, and } \\
\text { continued decrease in visual acuity (potentially permanent). }\end{array}$ \\
\hline V & Cavernous sinus thrombosis & Fevers, sepsis, and the development of cranial nerve palsies. \\
\hline
\end{tabular}


frontal sinusitis [31, 32]. Any of the classic meningeal signs can be associated with intracranial involvement, including photophobia, nuchal rigidity, hypertension, bradycardia, nausea, vomiting, and altered mental status. Specific pathogens associated with orbital and intracranial complications are the same as those associated with ARS. Treatment of brain abscess involves antibiotic therapy with high CNS penetration, as well as emergent surgical intervention by a neurosurgeon.

\section{Treatment algorithm for acute and chronic rhinosinusitis}

Antibiotics are the most commonly prescribed treatment for acute bacterial rhinosinusitis in children. There have been multiple studies performed evaluating efficacy of specific treatments in the pediatric population. Meta-analysis involving over 370 children shows significant reduction in symptoms and a higher rate of cure when compared to placebo in patients with symptoms of ARS more than 7-10 days [31, 32]. Uncomplicated ARS in a child should be treated with amoxicillin or, if extended spectrum is desired, amoxicillin/ clavulanate and cephalosporins can be used for coverage of beta-lactamase organisms $[25 \bullet \bullet]$.

In addition to antibiotic therapy, intranasal corticosteroid sprays offer improved recovery and symptom control in pediatric ARS [33, 34]. Several pediatric and adult trials have shown that intranasal steroids with antibiotic treatment offer benefit to cough and nasal discharge. Initiating treatment with antibiotics and intranasal steroids can be done comfortably by the primary care physician or pediatrician. In terms of additional therapy including decongestants, antihistamines, and nasal irrigation in children, the recently published EPOS consensus reviewed over 402 articles and concluded no evidence to determine whether these agents are efficacious in children [13]. Short courses of oral steroids can be of use especially in patients with pCRS with nasal polyps, severe allergies, or those with pulmonary sequela from sinusitis. Again, the management of most cases of acute rhinosinusitis can be carried out without the need for otolaryngology consultation, with referral of those cases that do not respond to medical therapy.

Specific treatment algorithms for pCRS are more complex owing to the multifactorial pathogenesis of the disease. While antimicrobial therapy is
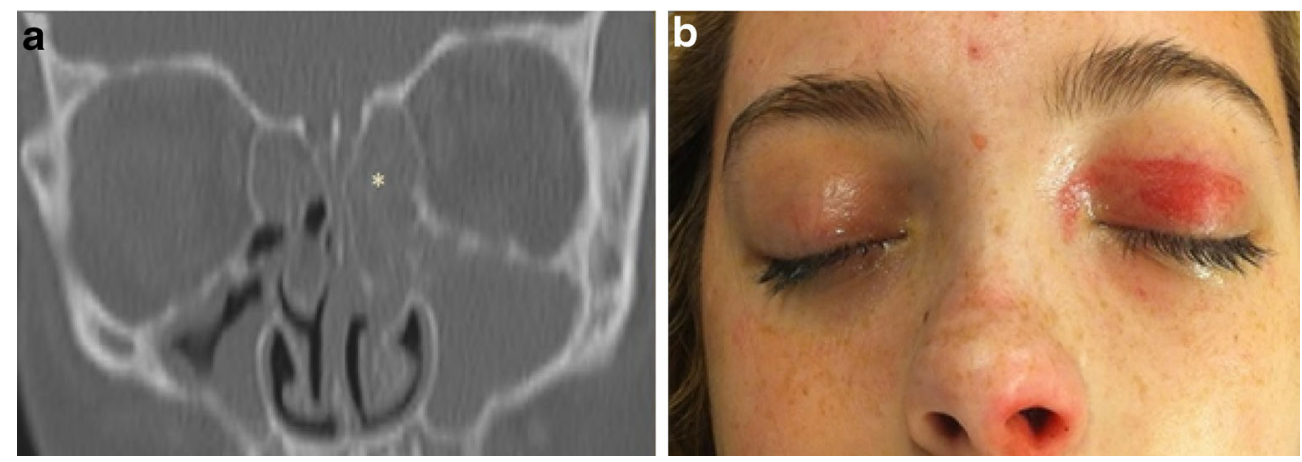

Fig. 1. Preseptal cellulitis. a CT findings of a 13-year-old female with acute sinusitis and left preseptal cellulitis. Asterisk, left ethmoid sinus. b The same patient's associated physical exam finding consistent with preseptal cellulitis 
important when there is an acute symptom exacerbation due to infection, it is important to also consider and address chronic inflammation and ostial obstruction. After having failed primary medical management, consultation to an otolaryngologist may be considered for cultures, endoscopic examination, or computed tomography (CT) scan. With regard to antibiotic treatment regimens, extended duration antibiotic therapy (at least 2-3 weeks, as opposed to 10 days) may produce improved and sustained clinical response [1]. Further, culture-directed antibiotic selection is always advised, especially in individuals who have failed empiric treatment. Similar to findings in ARS, topical nasal steroids have been shown to increase symptom resolution and control in patients with CRS. Nasal saline irrigation has also been shown to improve Lund-Mackay scores (as measured by the amount of CT sinus opacification)

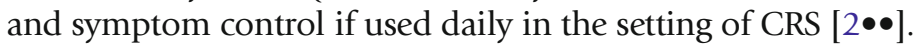

A number of comorbid diseases are seen in the setting of pCRS. Allergic rhinitis is often associated with CRS and thought to play part in the development and persistence of symptoms. There is currently no definite relationship detailed between allergic rhinitis and pCRS. A study evaluating over 351 children found a prevalence of positive allergy testing in $30 \%$ of children with CRS, with $32 \%$ of the general pediatric population testing positive for allergy (without CRS) [33]. While allergy and pCRS produce many similar symptoms, any cause-and-effect relationship is currently unknown.

More recently, there has been growing literature in the pediatric population suggesting a correlation between gastroesophageal reflux disease (GERD) and the development of CRS. In a review of 1980 children, a concomitant diagnosis of GERD and CRS was significantly higher than control (4.19\% compared to $1.35 \%$, respectively) [34]. Despite evidence of association, there is currently not enough evidence to recommend treatment of GERD for all pediatric patients with CRS, and neither the European consensus nor the AAO consensus have suggested such therapy $[2 \bullet \bullet, 13]$.

Competence of the immune system has also been implicated as a potential cause for pediatric rhinosinusitis. Immune deficiencies are present on $0.5 \%$ of the pediatric population with pCRS $[35,36]$. Humoral immunity matures to an adult level by approximately age 7 years, and children with a history of frequent bacterial infections or persistent sinus symptoms despite treatment should be referred for a thorough evaluation. Many studies have evaluated the humoral response in these patients and often show a decrease in specific immunoglobulin levels $[35,36]$. The most common immune deficiencies in the pediatric population are common variable, immunoglobulin G subclass, and selective antibody deficiency. In general, for patients with pCRS refractory to standard medical or surgical therapy, immunologic referral and assessment is warranted.

\section{Chronic rhinosinusitis in cystic fibrosis and ciliary dysfunction}

Cystic fibrosis (CF) is a genetic condition most often caused by a deletion of the amino acid phenylalanine at the 508th position of the cystic fibrosis transmembrane conductance regulator (CFTR) gene on chromosome 7q31.2. Wildtype CFTR acts as a chloride ion channel involved in sweat, digestion, and production mucus [37]. Mutations in this channel result in improper salt handling and thickening of secretions. These patients are known to develop 
early and refractory chronic rhinosinusitis and are plagued with tenacious mucus production. In fact, children who present with severe nasal polyps are recommended to have an evaluation for $\mathrm{CF}[2 \bullet \bullet]$. Inspissated secretions act as reservoirs for bacteria and represent a nidus for inflammation and recalcitrant infections. Management of sinus disease in a patient with cystic fibrosis should be done with the aid of an otolaryngologist, and referral should be initiated in the early stages of sinus disease for optimal medical and surgical management.

In surgical specimens taken from patients with cystic fibrosis, the bacteria most commonly isolated in culture include Pseudomonas aeruginosa, Staphylococcus aureus, and Streptococcus viridans [38]. There is also a statistically significant association between sinus cultures and lower airway lavage cultures for both Pseudomonas and S. aureus [38]. Multiple studies have affirmed this notion that, in CF patients, the paranasal sinuses act as a bacterial reservoir and occasionally seed the lower airway causing chronic lung infection and increased pulmonary symptoms [39]. In general, patients with CF require a multidisciplinary approach to their care with pulmonologists, otolaryngologists, and infectious disease specialists working closely together to manage surgical and medical issues.

Medical management of sinus disease in patients with CF is founded on the basic treatment of pCRS; however, differences in microbial flora must be considered. Further, while routine antibacterial sinus rinse is not recommended for all patients with pCRS, targeted antimicrobial lavage or inhaled nebulized solution has been shown to be beneficial after endoscopic sinus surgery (ESS) in patients with CF. Some commonly used compounded solutions include tobramycin, gentamycin, meropenem, and colistin for drug-resistant Pseudomonas. In some cases, aggressive surgical debridement and mechanical removal of inspissated mucus with postoperative aggressive medical therapy can provide CF patients with CRS equivalent postoperative improvement when compared to non-CF patients [40].

Primary ciliary dyskinesia (PCD) is an autosomal recessive disorder present in 1 of 15,000 people [41]. These patients suffer from a variety of microstructural abnormalities in cilia that give rise to the observed phenotype of ciliary dyskinesia. When a patient presents with symptomatology similar to cystic fibrosis (atypical asthma, bronchiectasis, chronic mucus production) but a negative CF test battery, evaluation for PCD should be considered. Treating the sinus manifestations of this disease is very similar to that of cystic fibrosis and includes aggressive irrigation regimens, culture-directed antibiotics, and endoscopic sinus surgery.

\section{Adenoidectomy in CRS}

The adenoids have been found to harbor microbial biofilms and thought to play in part to the seeding of the paranasal sinuses in patients with chronic rhinosinusitis. While further investigation into the exact role of the development and perpetuation of CRS is warranted, adenoidectomy can improve symptoms in up to $50 \%$ of children with CRS [42]. Again, the exact mechanisms behind this improvement are not clear. For this reason, the current AAOHNS consensus guidelines recommend adenoidectomy as an effective surgical procedure for children up to 12 years of age with CRS. Adenoidectomy alone 
can have a beneficial effect independent of ESS, especially in younger children. This treatment, combined with of medical therapy can offer an effective means of treatment for pCRS $[2 \bullet \bullet]$.

\section{Endoscopic sinus surgery for pediatric CRS}

ESS is a safe and highly effective treatment modality reserved for children that have failed maximum medical therapy [43]. Failure of medical therapy is difficult to define and often varies with each practitioner; however, it can loosely be defined by persistent symptoms despite the utilization of culture-directed antibiotics, intranasal steroid sprays, and saline irrigations for a period of at least 8 to 12 weeks. Current consensus released by the AAO has stated that ESS is an effective modality that is best performed when medical therapy and/or adenoidectomy have failed. Reduction in symptoms after ESS is as high as 82$100 \%$ with very low complication rates [44]. The approach to ESS in a pediatric patient is generally as conservative as possible, with great care to avoid mucosal stripping to preserve normal mucociliary pathways. In general, a majority of disease will be isolated to the anterior ethmoid and maxillary sinuses, which can be addressed simply and safely. The surgery will generally include removing the uncinate process, identifying then enlarging the maxillary sinus ostium. Debris, purulence, or polyps within the sinus can then be sampled then irrigated until clear. The ethmoid bulla is removed and the mucosa of the ethmoid sinuses inspected. If the mucosa is normal, the surgery is completed. This "miniFESS" approach allows maximal conservation of mucosa with adequate enlargement of the anterior paranasal sinuses.

Critics of ESS in pCRS often cite the concerns regarding preoperative imaging, need for repeat surgical debridement, and potential for facial growth derangements. Preoperative imaging is necessary prior to surgical intervention to allow for evaluation of the sinus anatomy and investigation into extent of disease. This also allows the utilization of intraoperative image guidance in patients with extensive polyposis or complicated anatomy. Multiple studies have shown that with the use of dissolvable packing, steroid nasal sprays, and modern surgical techniques, the success of ESS is no longer dependent on second-look procedures $[40,45]$. Revision sinus surgery is relatively uncommon and ranges from 13 to $39 \%$ of patients [46, 47]. Close follow-up and diligent use of postoperative medical therapy is essential for long-term results in most patients with CRS. There are some concerns about surgery affecting facial development. Studies on children after ESS with follow-up times ranging from 6 to 13 years that have reported no aberrancies in facial growth [48-50].

\section{Balloon catheter sinuplasty in the pediatric population}

Balloon catheter sinuplasty (BCS) is currently used as a treatment option for CRS in both pediatrics and adults. This procedure involves the trans-nasal placement of a small catheter into the patient's obstructed sinus ostium. The correct position of the catheter is then verified through direct visualization and transillumination. A balloon is then advanced over a guide wire, and a small balloon is inflated at the sinus ostium. Early results do confirm that BCS can safely be performed in the pediatric population, and the technology is currently 
approved by the US Federal Drug Administration for the treatment of pediatric sinusitis. The European Position Paper of Rhinosinusitis released a consensus that $\mathrm{BCD}$ can be utilized in the treatment algorithm in combination with adenoidectomy or other minimally invasive procedures [13]. While often performed in clinic on adult patients, pediatric populations usually require anesthesia and utilization of the operating room. In a recently published review of pediatric patients who failed medical therapy and were subsequently treated with BCD, $80 \%$ reported symptomatic improvement [51]. Because of these findings, and lack of current studies comparing ESS to BCD, the AAO-HNS could not reach a consensus statement on the treatment.

\section{Compliance with Ethics Guidelines}

\section{Conflict of Interest}

The authors declare that they have no competing interests.

\section{Human and Animal Rights and Informed Consent}

This article does not contain any studies with human or animal subjects performed by any of the authors.

\section{References and Recommended Reading}

Papers of particular interest, published recently, have been

highlighted as:

•• Of major importance

1. Clement PA, Bluestone CD, Gordts F, et al. Management of rhinosinusitis in children: consensus meeting, Brussels, Belgium, September 13, 1996. Arch Otolaryngol Head Neck Surg. 1998;124:31-4.

2.• Brietzke SE, Shin JJ, Choi S, et al. Clinical consensus statement: pediatric chronic rhinosinusitis. Otolaryngol Head and Neck Surgery. 2014;151:54253.

The most recent consensus statement released by the American Academy of Otolaryngology on the treatment of pediatric rhinosinusitis.

3. Wald ER. Diagnosis and management of acute sinusitis. Pediatr Ann. 1988;17:629-38.

4. Wang DY, Wardani RS, Singh $\mathrm{K}$, et al. A survey on the management of acute rhinosinusitis among Asian physicians. Rhinology. 2011;49:264-71.

5. Lin SW, Wang YH, Lee MY, et al. Clinical spectrum of acute rhinosinusitis among atopic and nonatopic children in Taiwan. Int J Pediatr Otorhinolaryngol. 2012;76:70-5.

6. Hall GM. Embryology and abnormal anatomy of the maxillary sinus. Northwest Med. 1969;68:1010-1.

7. Bingham B, Wang RG, Hawke M, Kwok P. The embryonic development of the lateral nasal wall from 8 to 24 weeks. Laryngoscope. 1991;101:992-7.
8. Park IH, Song JS, Choi H, et al. Volumetric study in the development of paranasal sinuses by CT imaging in Asian: a pilot study. Int J Pediatr Otorhinolaryngol. 2010;74:1347-50.

9. Kristo A, Uhari M, Luotonen J, et al. Paranasal sinus findings in children during respiratory infection evaluated with magnetic resonance imaging. Pediatrics. 2003;111:e586-9.

10. Spaeth J, Krugelstein U, Schlondorff G. The paranasal sinuses in CT-imaging: development from birth to age 25. Int J Pediatr Otorhinolaryngol. 1997;39:25-40.

11. Jang YJ, Kim SC. Pneumatization of the sphenoid sinus in children evaluated by magnetic resonance imaging. Am J Rhinol. 2000;14:181-5.

12. Lee DH, Shin JH, Lee DC. Three-dimensional morphometric analysis of paranasal sinuses and mastoid air cell system using computed tomography in pediatric population. Int J Pediatr Otorhinolaryngol. 2012;76:1642-6.

13. Fokkens WJ, Lund VJ, Mullol J, et al. European position paper on rhinosinusitis and nasal polyps 2012. Rhinology Supplement 2012:3 p preceding table of contents, 1-298.

14. Wald ER, Applegate KE, Bordley $\mathrm{C}$, et al. Clinical practice guideline for the diagnosis and management of 
acute bacterial sinusitis in children aged 1 to 18 years. Pediatrics. 2013; 132:e262-80.

15. van den Broek MF, Gudden C, Kluijfhout WP, et al. No evidence for distinguishing bacterial from viral acute rhinosinusitis using symptom duration and purulent rhinorrhea: a systematic review of the evidence base. Otolaryngol Head Neck Surg. 2014;150:533-7.

16. Wald ER, Milmoe GJ, Bowen A, Ledesma-Medina J, Salamon N, Bluestone CD. Acute maxillary sinusitis in children. N Engl J Med. 1981;304:749-54.

17. Brook I. Bacteriology of acute and chronic ethmoid sinusitis. J Clin Microbiol. 2005;43:3479-80.

18. Hsin CH, Su MC, Tsao CH, Chuang CY, Liu CM. Bacteriology and antimicrobial susceptibility of pediatric chronic rhinosinusitis: a 6-year result of maxillary sinus punctures. Am J Otolaryngol. 2010;31:145-9.

19. Costerton JW, Stewart PS, Greenberg EP. Bacterial biofilms: a common cause of persistent infections. Science. 1999;284:1318-22.

20. Nazzari E, Torretta S, Pignataro L, Marchisio P, Esposito $\mathrm{S}$. Role of biofilm in children with recurrent upper respiratory tract infections. Eur J Clin Microbiol Infect Dis. 2014.

21. Davies D. Understanding biofilm resistance to antibacterial agents. Nat Rev Drug Discov. 2003;2:114-22.

22. Sanclement JA, Webster P, Thomas J, Ramadan HH. Bacterial biofilms in surgical specimens of patients with chronic rhinosinusitis. Laryngoscope. 2005; 115:578-82.

23. Zuliani G, Carlisle M, Duberstein A, et al. Biofilm density in the pediatric nasopharynx: recurrent acute otitis media versus obstructive sleep apnea. Ann Otol Rhinol Laryngol. 2009;118:519-24.

24. Zuliani G, Carron M, Gurrola J, et al. Identification of adenoid biofilms in chronic rhinosinusitis. Int J Pediatr Otorhinolaryngol. 2006;70:1613-7.

25.• Fokkens WJ, Lund VJ, Mullol J, et al. EPOS 2012: European position paper on rhinosinusitis and nasal polyps 2012. A summary for otorhinolaryngologists. Rhinology. 2012;50:1-12.

An extensive review for all aspects of rhinology including basis of disease and treatment of current conditions.

26. Conrad DA, Jenson HB. Management of acute bacterial rhinosinusitis. Curr Opin Pediatr. 2002;14:86-90.

27. Stokken J, Gupta A, Krakovitz P, Anne S. Rhinosinusitis in children: a comparison of patients requiring surgery for acute complications versus chronic disease. Am J Otolaryngol. 2014;35:641-6.

28. Chandler JR, Langenbrunner DJ, Stevens ER. The pathogenesis of orbital complications in acute sinusitis. Laryngoscope. 1970;80:1414-28.

29. Garcia GH, Harris GJ. Criteria for nonsurgical management of subperiosteal abscess of the orbit: analysis of outcomes 1988-1998. Ophthalmology. 2000;107:1454-6. discussion 1457-1458.

30. Capra G, Liming B, Boseley ME, Brigger MT. Trends in orbital complications of pediatric rhinosinusitis in the United States. JAMA Otolaryngol Head Neck Surg. 2015;141:12-7.
31. Harris LF, Haws FP, Triplett Jr JN, Maccubbin DA. Subdural empyema and epidural abscess: recent experience in a community hospital. South Med J. 1987;80:1254-8.

32. Dolan RW, Chowdhury K. Diagnosis and treatment of intracranial complications of paranasal sinus infections. J Oral Maxillofac Surg. 1995;53:1080-7.

33. Leo G, Piacentini E, Incorvaia C, Consonni D, Frati F. Chronic rhinosinusitis and allergy. Pediatr Allergy Immunol. 2007;18 Suppl 18:19-21.

34. El-Serag HB, Gilger M, Kuebeler M, Rabeneck L. Extraesophageal associations of gastroesophageal reflux disease in children without neurologic defects. Gastroenterology. 2001;121:1294-9.

35. Costa Carvalho BT, Nagao AT, Arslanian C, et al. Immunological evaluation of allergic respiratory children with recurrent sinusitis. Pediatr Allergy Immunol. 2005;16:534-8.

36. Shapiro GG, Virant FS, Furukawa CT, Pierson WE, Bierman CW. Immunologic defects in patients with refractory sinusitis. Pediatrics. 1991;87:311-6.

37. Kerem E. Atypical CF, and CF related diseases. Paediatr Respir Rev. 2006;7 Suppl 1:S144-6.

38. Godoy JM, Godoy AN, Ribalta G, Largo I. Bacterial pattern in chronic sinusitis and cystic fibrosis. Otolaryngol Head Neck Surg. 2011;145:673-6.

39. Hansen SK, Rau MH, Johansen HK, et al. Evolution and diversification of Pseudomonas aeruginosa in the paranasal sinuses of cystic fibrosis children have implications for chronic lung infection. ISME J. 2012;6:31-45.

40. Duplechain JK, White JA, Miller RH. Pediatric sinusitis. The role of endoscopic sinus surgery in cystic fibrosis and other forms of sinonasal disease. Arch Otolaryngol Head Neck Surg. 1991;117:422-6.

41. Sleigh MA. Primary ciliary dyskinesia. Lancet. 1981;2:476.

42. Brietzke SE, Brigger MT. Adenoidectomy outcomes in pediatric rhinosinusitis: a meta-analysis. Int J Pediatr Otorhinolaryngol. 2008;72:1541-5.

43. Vlastarakos PV, Fetta M, Segas JV, Maragoudakis P, Nikolopoulos TP. Functional endoscopic sinus surgery improves sinus-related symptoms and quality of life in children with chronic rhinosinusitis: a systematic analysis and meta-analysis of published interventional studies. Clin Pediatr. 2013;52:1091-7.

44. Lieser JD, Derkay CS. Pediatric sinusitis: when do we operate? Curr Opin Otolaryngol Head Neck Surg. 2005; 13:60-6.

45. Mitchell RB, Pereira KD, Younis RT, Lazar RH. Pediatric functional endoscopic sinus surgery: is a second look necessary? Laryngoscope. 1997;107:1267-9.

46. Ramadan HH. Revision endoscopic sinus surgery in children: surgical causes of failure. Laryngoscope. 2009;119:1214-7.

47. Lee TJ, Liang CW, Chang PH, Huang CC. Risk factors for protracted sinusitis in pediatrics after endoscopic sinus surgery. Auris Nasus Larynx. 2009;36:655-60. 
48. Mair EA, Bolger WE, Breisch EA. Sinus and facial growth after pediatric endoscopic sinus surgery. Arch Otolaryngol Head Neck Surg. 1995;121:547-52.

49. Bothwell MR, Piccirillo JF, Lusk RP, Ridenour BD. Long-term outcome of facial growth after functional endoscopic sinus surgery. Otolaryngol Head Neck Surg. 2002;126:628-34.
50. Senior B, Wirtschafter A, Mai C, Becker C, Belenky W. Quantitative impact of pediatric sinus surgery on facial growth. Laryngoscope. 2000;110:1866-70.

51. Ramadan HH, Terrell AM. Balloon catheter sinuplasty and adenoidectomy in children with chronic rhinosinusitis. Ann Otol Rhinol Laryngol. 2010;119:578-82. 